\title{
1 Tiara: Deep learning-based classification system for eukaryotic sequences
}

3 Michał Karlicki ${ }^{1}$, Stanisław Antonowicz ${ }^{1}$, Anna Karnkowska ${ }^{1}$

4

$5{ }^{1}$ Institute of Evolutionary Biology, Faculty of Biology, Biological and Chemical Research Centre,

6 University of Warsaw, Warsaw, Poland

Corresponding author:

9 Anna Karnkowska, Institute of Evolutionary Biology, Faculty of Biology, Biological and Chemical

10 Research Centre, University of Warsaw, Żwirki i Wigury 101, Warsaw, ZIP: 02 089, Poland,

11 a.karnkowska@uw.edu.pl, Phone: +48 225526641.

12

\section{Abstract}

14 Motivation:

15 With a large number of metagenomic datasets becoming available, the eukaryotic metagenomics 16 emerged as a new challenge. The proper classification of eukaryotic nuclear and organellar genomes 17 is an essential step towards the better understanding of eukaryotic diversity.

18 Results:

19 We developed Tiara, a deep-learning-based approach for identification of eukaryotic sequences in 20 the metagenomic data sets. Its two-step classification process enables the classification of nuclear 21 and organellar eukaryotic fractions and subsequently divides organellar sequences to plastidial and 22 mitochondrial. Using test dataset, we have shown that Tiara performs similarly to EukRep for prokaryotes classification and outperformed it for eukaryotes classification with lower calculation

24 time. Tiara is also the only available tool correctly classifying organellar sequences.

25 Availability and implementation:

Tiara is implemented in python 3.8, available at https://github.com/ibe-uw/tiara and tested on Unixbased systems. It is released under an open-source MIT license and documentation is available at https://ibe-uw.github.io/tiara. Version 1.0.1 of Tiara has been used for all benchmarks. 


\section{Introduction}

Microbial communities of unicellular eukaryotes (protists) and prokaryotes are an essential part of all ecosystems. Next to prokaryotes, protists are significant drivers in diverse nutrient cycling pathways. Autotrophic and mixotrophic protists fix carbon in aquatic environments, whereas heterotrophic protists catalyze nutrient cycling in aquatic and terrestrial ecosystems as selective consumers of bacteria and fungi (Caron et al., 2009).

Metagenomic studies changed our understanding of the prokaryotic communities and allowed us to uncover their taxonomic and functional diversity in various environments (Sunagawa et al., 2015; Almeida et al., 2019). However, even though microeukaryotes are key components of microbial communities, their study lags behind the study of prokaryotes (Keeling and Campo 2017), and that is particularly true for the metagenomic studies. Until now, mainly metabarcoding (e.g. De Vargas et al., 2015), metatranscriptomics (e.g. Salazar et al., 2019), and single-cell genome sequencing (e.g. Strassert et al., 2018) were used to explore the diversity of microbial eukaryotes. In contrast to these methods, the utilization of the metagenomic approaches was hampered by the complexity and size of eukaryotic genomes, as well as a limited number of reference databases allowing further taxonomical or functional annotation. With a few exceptions, such as phytoplankton (Delmont et al., 2015; Duncan et al., 2020) or human microbiome (Olm et al., 2019) studies, eukaryotes were neglected in metagenomic studies. Only recently the metagenomic datasets from large sampling projects, such as the Tara Oceans expedition (Pesant et al., 2015) or Ocean Sampling Day (Kopf et al., 2015), were exploited to uncover the eukaryotic plankton biogeography (Richter et al., 2019; Leconte et al., 2020), taxonomy (Obiol et al., 2020), and functional diversity (Delmont et al., 2020). Metagenomic data often do not contain a sufficient amount of data to reconstruct nuclear genomes, but mitochondrial and plastid genomes, owing to their smaller size and a higher number of copies, may be potentially reconstructed from those data. Most often the mitochondrial genomes (Andújar et al., 2015; Crampton-Platt et al., 2016) or only single genes, such as 16S rDNA (Piganeau and Moreau, 2007; Piganeau et al., 2008), are reconstructed from the metagenomic data. Organellar genomes have been shown to provide suitable data to address questions about microbial eukaryotes' evolution and ecology (Cuvelier et al., 2010; Kim et al., 2011; Wideman et al., 2020). However, organellar data are mostly unexplored in the metagenomes, since they are often classified as bacterial sequences, and are thus removed from the eukaryotic genome assemblies (Delmont et al., 2020; Duncan et al., 2020). Only a few approaches dedicated to the processing of the eukaryotic fraction from the metagenomic data exist. They might be split into those developed to analyse raw reads (Wood et al., 2019) or single genes (Schön et al., 2020), both of which strongly depends on the reference databases. 
Alternatively, the eukaryotic nuclear genomes might be reconstructed from the data using one of the two main existing pipelines. In the first approach, the assembled contigs are binned, visualized, and manually refined using Anvio'O (Eren et al., 2015; Delmont and Eren, 2016), whereas the second approach, used in EukRep, assumes initial separation of contigs into two domains (Prokarya and Eukarya), and then binning within those two groups independently (West et al., 2018). Both approaches have been successfully used for obtaining partial nuclear eukaryotic genomes but failed to correctly classify the organellar fraction in the metagenomic data (Duncan et al., 2020; Delmont et al., 2020). Only one tool, MitoZ, was designed explicitly for the organellar data, but it is only applicable for the assembly, identification and analysis of the animals' mitochondrial genomes (Meng et al., 2019).

The most widely used tools for biological sequence comparison are alignment-based methods such as Smith-Waterman algorithm (Smith and Waterman, 1981) and its further developments such as BLAST (Altschul et al., 1990) or BLAT (Kent, 2002). Several binning algorithms relying on the alignment-based approach, such as taxator-tk (Dröge et al., 2015), have been proposed for the taxonomic assignment of DNA sequences in metagenomes. Although the alignment-based methods are the most accurate for sequence comparisons, they fail if sequences are highly divergent or the reference database is limited. These methods are also computationally intensive for large datasets (Ren et al., 2018). For those reasons, the use of alignment-based methods for metagenomic data is relatively confined. Alignment-free methods, based on $k$-mers or DNA substrings, provide promising alternatives to overcome the weaknesses of alignment-based methods (Ren et al., 2018). The usage of alignment-free methods is currently rapidly growing, and especially machine learning approaches have been used extensively for classification of various types of sequences from metagenomes (Liang et al., 2020; Krawczyk et al., 2018). The most potent approaches are based on deep learning, a family of machine learning methods based on artificial neural networks. Those methods best exploit large and multidimensional data sets and tackle intricate patterns in the data (Angermueller et al., 2016).

The most broadly used tool for the eukaryotic metagenomics is EukRep, which uses $k$-mer frequencies and linear SVMs for DNA sequences classification (West et al., 2018). It was shown to be useful for obtaining high quality nuclear eukaryotic genomes from complex environmental samples (West et al., 2018), but lacks features which would enable proper organellar genomes classification. Here, we introduce Tiara, a deep-learning-based approach for identification of eukaryotic sequences in the metagenomic data sets. Its two-step classification process enables to classify nuclear and organellar eukaryotic fractions and subsequently divide organellar data into plastid and mitochondrial. Tiara outperforms EukRep in terms of prediction accuracy and calculation time. 
bioRxiv preprint doi: https://doi.org/10.1101/2021.02.08.430199; this version posted February 9, 2021. The copyright holder for this preprint (which was not certified by peer review) is the author/funder, who has granted bioRxiv a license to display the preprint in perpetuity. It is made available under aCC-BY-ND 4.0 International license.

\section{Methods}

In the first step, Tiara classifies assembled DNA sequences (contigs) into six classes: Archaea, Bacteria, Prokarya (either Bacteria or Archaea but with no specific classification), Eukarya, organelles, and unknowns (Fig. 1, the first step of classification). In the subsequent step, the organellar sequences are further classified into plastids, mitochondria, and unknowns (Fig. 1, the second step of classification). For the classification, the method employs two feed-forward neural networks and $k$ mer compositions.

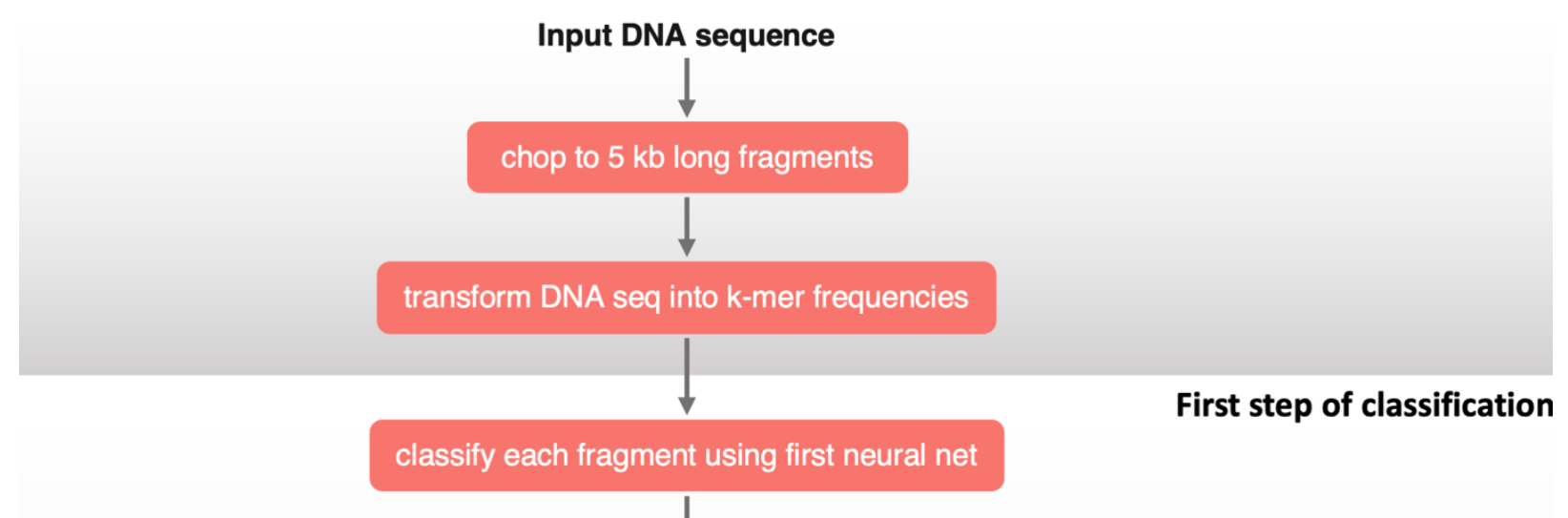

take the mean across all classes

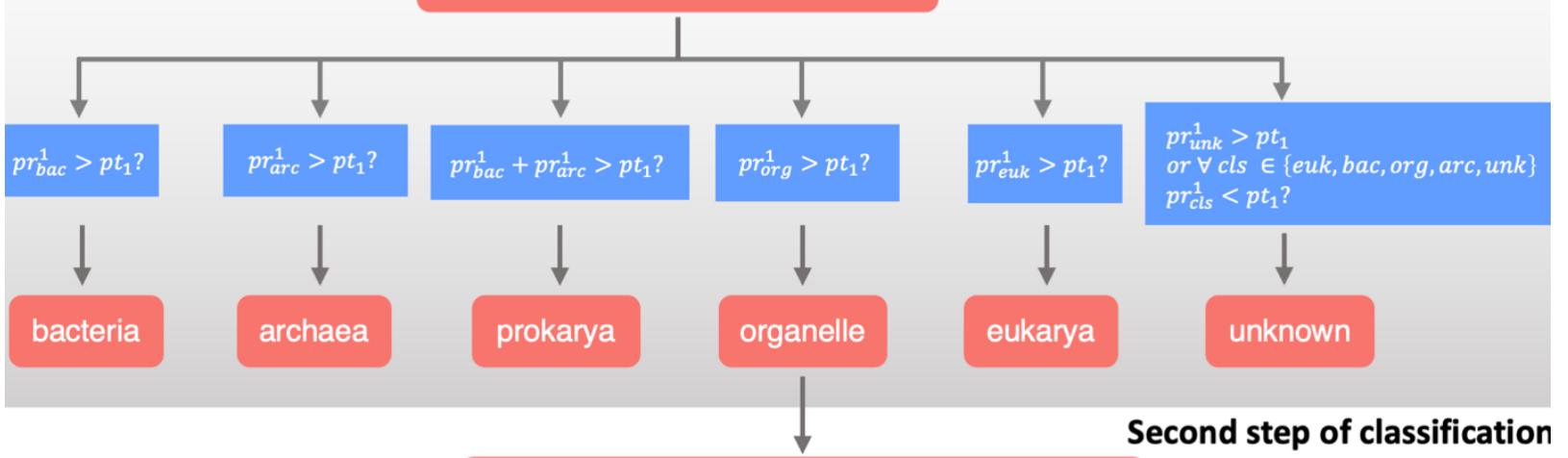

classify each fragment using second neural net

take the mean across all classes

Figure. 1. Scheme of the main steps of dataflow implemented in Tiara. 


\subsection{Training and test datasets}

109 We decided to take advantage of taxonomic information to emerge well balanced and not

110 overlapping train and test datasets. We independently picked genomes for each category to obtain a

111 diverse final dataset despite differences in genomes' lengths and their representation in reference

\section{2 databases.}

113 Training datasets were prepared based on 8,220 genomic sequences (Supplementary Table S1) from 114 three domains of life: Eukarya (4381) [nuclear (73), plastid (2260), and mitochondrial genomes

115 (2048)], Bacteria (1860) and Archaea (1979). Data were downloaded from the NCBI Genome

116 database (Agarwala et al., 2018) and the Joint Genome Institute (JGI) (Grigoriev et al., 2012).

117 Eukaryotic nuclear genomes were chosen manually and included genomes from large groups of

118 eukaryotes representing all supergroups, i.e., Stramenopiles, Alveolates, Chlorophyts, Rhodophytes,

119 Amoebozoa, Opisthokonta and Discoba). Mitochondrial genomes from the NCBI genome database

120 marked as: "Fungi", "Plants", "Protist" and "Other" were selected. In the case of animal

121 mitochondrial genomes, we selected only those marked as "Other Animals" and "Insects" as

122 representatives of this large group of small and homogeneous genomes. For plastid representation,

123 we downloaded all available plastid genomes annotated as "Green algae", "Protist" and "Other" in

$124 \mathrm{NCBI}$, and one representative per each genus of Land Plants to avoid overrepresentation of plants'

125 plastid genomes. In the case of bacterial genomes, we selected one representative from each genus

126 present in the NCBI Genome with the best assembly quality. Due to the insufficient number of

127 complete archaeal genomes and a dominant number of low-quality genomes derived from

128 metagenomic initiatives, we used the best quality genomes for each archaeal species present in the

129 NCBI genome database. Subsequently, all genome sequences were split into $5 \mathrm{~kb}$ fragments, and for

130 bacterial genomes, $10 \%$ of fragments of each genome were randomly picked to achieve the better

131 class balance between eukaryotes and prokaryotes. Fragments containing other letters than $\{A, T, G$,

132 C $\}$ were filtered out. Subsequently, we selected a subset of the training set as a test set, and those

133 genomes were removed from the training set.

134 To test Tiara and compare it with EukRep, we prepared a test set based on 400 genomic sequences.

135 Genomes selected as test set are not present in EukRep training dataset nor the Tiara training set

136 and were selected with maximum overlap with the training genomes set at the genus level. The test

137 set contains 165 eukaryotic genomes (105 nuclear, 28 plastidial and 32 mitochondrial) and 385

138 prokaryotic genomes (306 Bacteria and 79 Archaea). We supplemented the test dataset with data

139 from large eukaryotic and prokaryotic groups (such as Cryptophyta, Haptophyta and

140 Glaucocystophyta in case of eukaryotes and Candidate Phyla Radiation group, in case of prokaryotes)

141 not represented in training set to test the Tiara ability of proper classification of divergent genomic 
sequences. Genomes were downloaded from NCBI and JGI databases (Supplementary Table S2). The genomes with less than 20 contigs were chopped into $100 \mathrm{~kb}$ long chunks, and less contiguous

144 assemblies remained unchanged to reflect the condition of metagenomic assemblies. Mitochondrial and plastidial genomes were fragmented into pieces in a range of $1 \mathrm{~kb}-75 \mathrm{~kb}$ (Supplementary Methods).

\subsection{Sequence representation}

We used tf-idf (tf - term frequency, idf - inverse document frequency) - a technique used in information retrieval - to represent DNA sequences. Each sequence was represented as a real-valued vector of length $4^{k}$, where $k$ was the $k$-mer length. Given a set of sequences $\mathcal{S}$ and $s \in \mathcal{S}$, we defined $t f_{s}$ as the oligonucleotide frequency vector for a sequence $s$ and $i d f_{\mathcal{S}}$ as a vector describing the inverse document frequency of each $k$-mer:

$$
i d f_{\mathcal{S}}^{i}=\log \left(\frac{|\mathcal{S}|+1}{d(\mathcal{S}, i)+1}\right),
$$

where $d(\mathcal{S}, i)$ is equal to the number of sequences $s \in \mathcal{S}$ that contain $i$-th $k$-mer (in the lexicographic order). Then the representation of the sequence $s$ was calculated as: $v_{s}=t f_{s} \otimes i d f_{\mathcal{S}}$, where $\otimes$ is the piecewise multiplication of vectors. The vectors are then normalized to sum to one. The effect of this representation is that $\mathrm{k}$-mers that occur in many DNA fragments weigh less to the prediction compared to k-mers present in only a few DNA fragments.

160 We have written our version of oligonucleotide frequency $\left(t f_{s}\right)$ calculation and an $i d f_{S}$ vector

161 calculation method that works online (processing one sequence at a time).

\subsection{Classification system}

163 We employed a two-stage classification method. In the first stage, the input sequences are classified

164 into six classes: bacteria, archaea, prokaryote, eukaryote, organelle or unknown. The second stage

165 differentiates between organelle subclasses: mitochondria, plastids, and unknown (Figure 1). This

166 two-stage process relies on the two distinct two-layer feed-forward neural network architectures.

167 Hyperparameter selection and training procedure are described in subsection 2.4.

168 During the classification process, we split the sequences into smaller fragments (5 kb). We then

169 classify each fragment separately and take the mean probability for each class, resulting in five (in

170 the first step of classification) or three (in the second step) values. We use $\operatorname{pr}_{\text {class }}^{i}$ notation to

171 describe the mean output of the neural network at stage $i$ for a specific class. The classification is

172 performed based on the probability thresholds $p t_{i}, i \in\{1,2\}$, one for each classification stage. The

173 sequence at stage $i$ is classified to a class if $p r_{\text {class }}^{i}>p t_{i}$. The exception from this rule is the situation 
174 where $p r_{\text {archaea }}^{1}<p t_{1}$ and $p r_{\text {bacteria }}^{1}<p t_{1}$, but $p r_{\text {archaea }}^{1}+p r_{\text {bacteria }}^{1}>p t_{1}$. The sequence is

175 then classified to a more general prokaryota class. If none of the above conditions are met, the

176 sequence is classified to an unknown class.

\section{$177 \quad 2.4$ Neural network architectures}

\section{Training}

179 We implemented and trained our models using PyTorch (Paszke et al., 2019) and skorch (Tietz et al., 180 2017) packages using negative log-likelihood loss and an Adam optimizer (Kingma and Ba, 2015). We 181 split the data into training (90\%) and validation (10\%) sets using a stratified splitting strategy: in each 182 set, the proportion of sequences from each class was the same. The batch size was set to 128.

183 Validation dataset was used to determine the best model.

185 To choose the best architectures we performed a search over several hyperparameters: lengths of kmers, number of nodes in the first and second neural network layer, dropout probability, learning rate and the number of epochs. We used several metrics to compare the models: accuracy, mean precision, mean recall and mean F1 score (Supplementary Methods). The means were taken across all classes. We evaluated the models on a validation set with a probability threshold of 0.5. For consistency, we picked the architecture with the highest average mean F1 score across all learning epochs. The results of the search are in Supplementary Tables S3 and S4. To choose the architectures

192 for the first stage, 15,900 hyperparameter combinations were tested, whereas for the second stage

19317,600 combinations were tested.

\subsection{Implementation and availability}

195 We developed our tool in Python 3.8, with use of the libraries skorch (Tietz et al., 2017), PyTorch

196 (Paszke et al., 2019), biopython (Cock et al., 2009), numba (Lam et al., 2015), joblib (Varoquaux and

197 Grisel, 2009) and tqdm. Tiara code is freely available under MIT license, and the code is stored on

198 GitHub (https://github.com/ibe-uw/tiara). The models used in the program by default are the best

199 models for each class (marked in bold in Supplementary Table S6), but the user can choose other

200 optimal models for each $k$-mer length. By default, Tiara returns tabular output with a class assigned

201 to each contig name but optionally allowing the user to output classified sequences to separate files 202 in fasta format.

\section{Results}

204 We implemented in Tiara a two-stage approach for classification of eukaryotic sequences from 205 assembled metagenomic data. In the first stage, Tiara classifies sequences into six categories 
206 (Archaea, Bacteria, Prokarya, Eukaryota, organelles or unknown). In the second stage, putative

207 organellar sequences are classified into plastids and mitochondria or unknown. Each stage of

208 classification encapsulates trained neural network model. Next, we evaluated its performance and

209 compared with EukRep using independent test dataset and showed usability using real metagenomic 210 data.

\section{$211 \quad 3.1$ Performance comparison of different $\boldsymbol{k}$-mer sizes}

212 We searched hyperparameter space to obtain the best neural network models (nearly 35000 models)

213 using a validation dataset. The best hyperparameters for each $k$-mer length and classification stage

214 for an optimal number of epochs are shown in Supplementary Table S5. The best first stage neural

215 network was trained with $k$-mer length 6, and had two layers with 2048 and 1024 nodes,

216 respectively. The best neural network in the second stage of classification used a $k$-mer length 7 and

217 had two layers with 128 and 64 nodes. For both stages, we used the dropout probability of 0.2 . The

218 first best model was trained for 41 epochs using a learning rate equal to 0.001 , and the second for 47

219 epochs with a learning rate of 0.01 . The comparison of the best models for each $k$-mer length (in

220 bold) with a sub-optimal architecture (both layer sizes equal to 32, learning rate of 0.01 and 0.5

221 dropout probability - in italics) shows that larger neural networks are necessary to identify the

222 biological signal present in the DNA sequences (Supplementary Table S5).

\section{3.2. Performance of trained models}

\section{Classification of eukaryotic and prokaryotic sequences}

225 Depending on the $k$-mer length, Tiara achieved on the test dataset mean prediction accuracy

226 between $98.65 \%$ and $98.93 \%$ for prokaryotic genomes and between $95.94 \%$ and $98.83 \%$ for the

227 nuclear genomes (Table 1). The best ratio between prediction accuracies for each class was noted for

$228 k$-mer 6 . Using this model, $96 \%$ of nuclear eukaryotic and $98 \%$ of prokaryotic genomes were classified

229 with higher or equal prediction accuracy to $90 \%$. Only three prokaryotic genomes have been

230 classified with accuracy lower than $50 \%$. However, most of the contigs derived from these genomes

231 were assigned as "unknown", and only two were classified as a eukaryote. All of these genomes were

232 small and highly reduced, and they belonged to symbionts or parasites. This bias was also previously

233 observed for EukRep (West et al., 2018). We checked probability outputs for them and observed

234 strong organellar signal for two endosymbionts, which might reflect the reductive evolution of their

235 genomes.

236 Importantly, Tiara achieved high accuracies (above 90\%) for genomes from groups of taxa that were

237 absent in the training dataset like eukaryotic haptophytes and cryptophytes or prokaryotic CPR, 
238 which indicates that our models are not overfitting despite employing complex neural networks.

239 Hence, Tiara will be able to classify contigs of novel evolutionary lineages correctly.

\section{Classification of organellar sequences}

241 Tiara achieved high prediction accuracy for a testing set of organellar genomes (28 plastidial and 32

242 mitochondrial) with an average accuracy above 95\% (Table 1). The best average accuracies were

243 observed for $k$-mer 6 (pt: 99.60\%; mt: 98.86\%). Similar to the nuclear genomes' classification, the

244 accuracy of organellar genomes classification increased with $k$-mer length. (Supplementary Table S6).

245 Analysis of fragmented organellar genomes (mitochondria: (1-5 kb) and plastids (1 kb-75 kb) for $k$ -

246 mers $k=\{4-6\}$ ) showed that prediction accuracy increased with the fragment length (Supplementary

247 Figure S1 and S2;). For organellar sequences longer than $3 \mathrm{~kb}$, accuracy was higher than 90\%, and for

248 sequences longer or equal to $5 \mathrm{~kb}$ - close to $100 \%$. In the second stage, most of the sequences were

249 assigned correctly to a given class (higher than 90\%) if the sequence was longer than $3 \mathrm{~kb}$.

250 Table 1. Comparison of accuracy for Tiara and Eukrep tools. All Tiara tests have been done by 251 default with 0.65 probability cutoffs. EukRep was tested with default settings. The best model for a 252 given class is shown in bold.

\begin{tabular}{ccccc}
\hline Software & $k$-mer & $\begin{array}{c}\text { Avg. accuracy Eukarya } \\
(\mathrm{n}=105)\end{array}$ & $\begin{array}{c}\text { Avg. accuracy } \\
\text { Prokarya }(\mathrm{n}=385)\end{array}$ & $\begin{array}{c}\text { Avg. accuracy } \\
\text { organelles }(\mathrm{n}=60)^{*}\end{array}$ \\
\hline Tiara & 4 & 0.9593 & 0.9871 & $0.9411^{\mathrm{mt}} / 0.9708^{\mathrm{pt}}$ \\
Tiara & 5 & 0.9641 & 0.9865 & $0.9841^{\mathrm{mt}} / 0.9981^{\mathrm{pt}}$ \\
Tiara & 6 & $\mathbf{0 . 9 8 8 3}$ & $\mathbf{0 . 9 8 9 3}$ & $\mathbf{0 . 9 8 8 6 ^ { \mathrm { mt } } / 0 . 9 9 6 ^ { \mathrm { pt } }}$ \\
EukRep & 5 & 0.963 & 0.9849 & $0.4738^{\mathrm{mt}} / 0.2979^{\mathrm{pt}}$
\end{tabular}

* In the case of EukRep we calculated the ratio of organellar fragments classified as Eukaryote.

\section{$255 \quad 3.3$ Performance comparison between Tiara and EukRep}

256 We compared Tiara with EukRep - a tool designed for the classification of eukaryotic and prokaryotic 257 sequences from metagenomic data (West et al., 2018). EukRep was previously shown to outperform 258 alignment-based methods. Thus, we compared Tiara only with EukRep, which is currently the state259 of-the-art method. Finally, it enabled the fast identification of eukaryotic contigs and further forming 260 eukaryotic MAGs (Metagenome Assembled Genomes). EukRep, similarly to Tiara, transforms DNA 261 sequences into $k$-mer frequencies, but then uses linear-SVM (implemented in scikit-learn) for 262 predictions, whereas Tiara uses sequential feed-forward neural networks. EukRep, as a binary 
bioRxiv preprint doi: https://doi.org/10.1101/2021.02.08.430199; this version posted February $9,2021$. The copyright holder for this preprint (which was not certified by peer review) is the author/funder, who has granted bioRxiv a license to display the preprint in perpetuity. It is made available under aCC-BY-ND 4.0 International license.

263 classifier, separates data only into two classes (domains): eukaryotes and prokaryotes. Therefore,

264 EukRep hasn't been trained on organellar DNA, so it was unclear how it classifies those sequences.

265 Tiara scored slightly better than EukRep, with the prediction accuracy of eukaryotic genomes $2.53 \%$

266 higher and prokaryotic genomes $-0.44 \%$ higher (Supplementary Table S2). We also calculated the

267 difference in prediction accuracy between Tiara and EukRep for each genomes pair (Fig. 2) to

268 examine prediction accuracy in details. For eukaryotic genomes (105), Tiara got better accuracies in

26963 cases, and only for 25 genomes, the results were worse. Whereas prokaryotes were classified

270 more evenly and for 275 genomes, both tools have the same results; in 89 cases, Tiara was better

271 than EukRep, and in 21 cases it was worse.

A
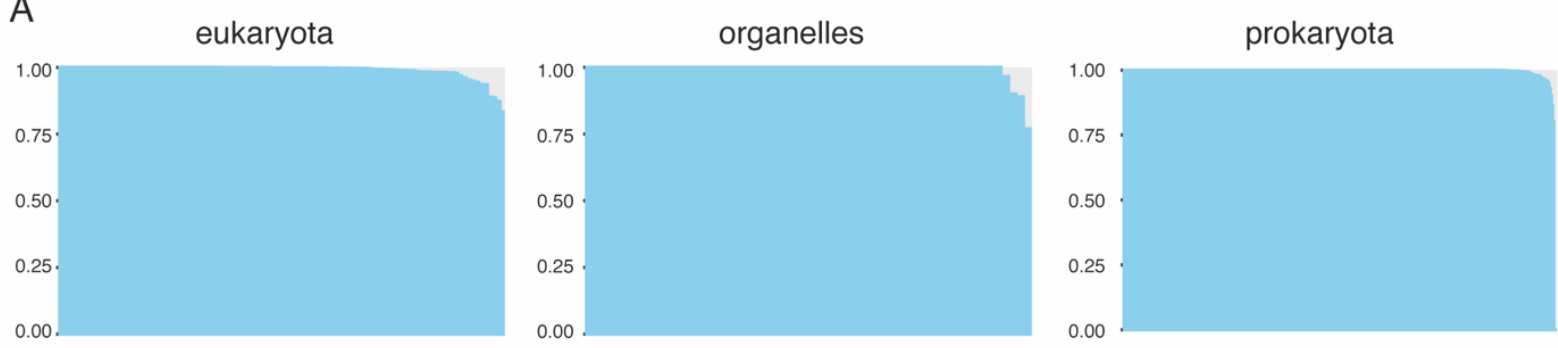

B eukaryota
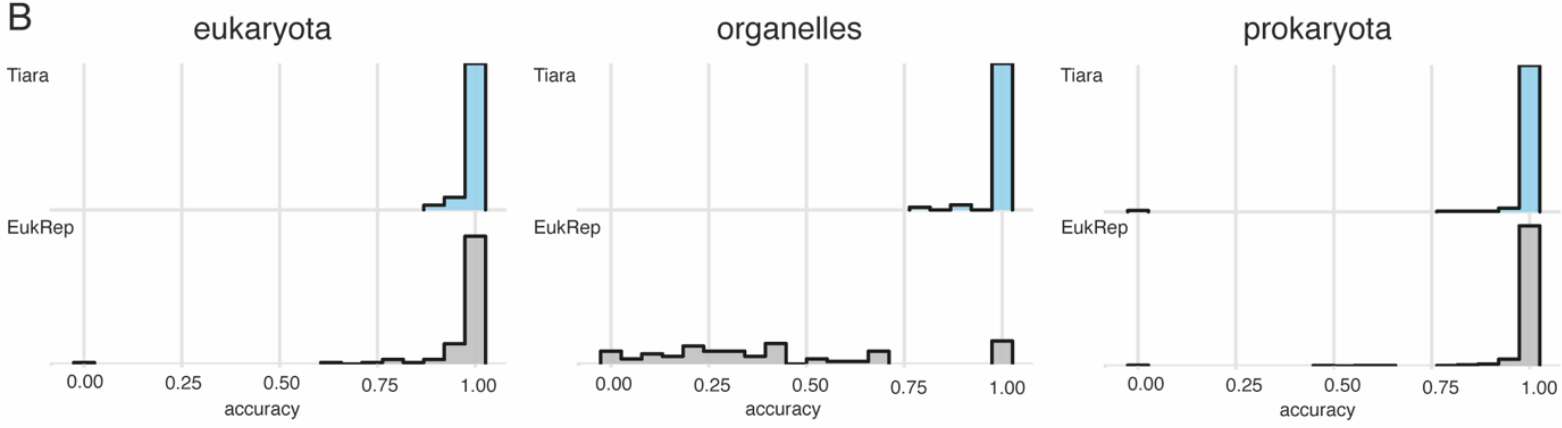

Figure. 2. Efficiency of Tiara classification and comparison to EukRep using a set of test genomes. Test genomes were divided into three groups: eukaryotic nuclear genomes (eukaryota), plastid and mitochondrial genomes (organelles), and archaeal and bacterial genomes (prokaryota). (A) Accuracy of Tiara for each genome in three groups (B) Histogram of the density of accuracy for EukRep and Tiara for three groups of genomes. All tests have been performed using the model with $k$-mer 6 and 0.65 probability cutoff.

280 To test the organellar genomes classification by EukRep, we used a set of fragmented plastid (10 kb)

281 and mitochondrial ( $5 \mathrm{~kb}$ ) genomes. EukRep assigned only $47 \%$ of mitochondrial and $30 \%$ of plastid

282 contigs as eukaryotic sequences (Supplementary Table S2).

283 Additionally, we checked the speed performance of both approaches (Supplementary Methods).

284 Tiara supports parallel execution, whereas EukRep uses all cores available. However, for one core,

285 Tiara was two times faster than EukRep. Finally, Tiara using 12 cores classified the test genome 
roughly five times faster than EukRep and reached a speed of $6.8 \mathrm{Mbp}$ per second. (Supplementary

287 Table S7).

\section{$288 \quad 3.4$ Classification of sequences from the real data}

289 To examine our approach on real metagenomic data, we used three datasets from the Tara Oceans

290 project (Pesant et al., 2015). We selected three samples from the Mediterranean Sea (ERR1726574,

291 ERR1726673, ERR868402) from the same station, representing three different size fractions

292 associated with protists (Supplementary Methods, Supplementary Table S8). Data were assembled

293 (Supplementary Methods; Table S8) and used for further analyses. We tested Tiara with three $k$-mer

294 lengths $(4,5$, and 6$)$ and three minimum sequence lengths $(1000,3000,5000 \mathrm{bp})$ for the first stage of

295 classification (Supplementary Methods).

Composition of metagenomic samples

In the metagenome of the smallest size fraction $(0.8-20 \mu \mathrm{m})$, prokaryotes seemed to prevail, as the majority of contigs were classified as Bacteria, Archaea or Prokarya (up to 97\% for $k=5$ ); however, datasets from larger size fractions (20-180 and 180-2000 $\mu \mathrm{m}$ ) were dominated by eukaryotes (up to $96 \%$ for $k=6$ ) (Supplementary Figure S3 and Table S9). Contribution of contigs assigned to eukaryotes was the highest using model with the $k$-mer length $\mathrm{k}=6$, which is in line with the results obtained

302 from the test datasets. The organellar fraction's overall contribution was low in assembled data and 303 ranged between $0.26 \%$ and $4.2 \%$ across datasets and analysis variants. Nevertheless, organellar 304 contigs were among the longest ones and exceeded $50 \mathrm{~kb}$ for sample ERR1726673 (Supplementary 305 Table S10).

\section{Analysis of large fragments classified as organelles}

307 We annotated 21 contigs assigned as organellar and longer than $10 \mathrm{~kb}$ (Supplementary Methods).

308 Among those 21 contigs, 13 were annotated as mitochondrial and seven as plastidial, and for one, 309 blastN reported no significant hits. (Supplementary Table S10). For the smallest size fraction (0.5-20 $310 \mu \mathrm{m})$, all five analyzed contigs were derived from two plastid genomes, belonging to the 311 dictyochophycean Florenciella parvula and the green alga Pycnococcus provasoli. Three fragments of 312 the Pycnococcus plastid genome together accounted for the $58.2 \%$ of its estimated size and carried 31338 genes. The largest taxonomic diversity of contigs was detected in the size fraction 20-180 $\mu \mathrm{m}$; 314 organellar genomes of nine protists (diatoms, ciliates) and animals (crustaceans, insects and 315 molluscs) were identified. For the largest size fraction $(180-2000 \mu \mathrm{m})$, we identified three partial 316 mitochondrial genomes that belonged to animals (crustaceans and hydrozoans). 


\section{Discussion}

319 We developed Tiara, a machine learning-based tool, which can efficiently and accurately separate eukaryotic sequences from the prokaryotic ones to overcome difficulties with eukaryotic data

321 classification in the metagenomic data. Tiara doesn't rely on large reference databases and can be efficiently utilized in pipelines for identifying eukaryotic scaffolds and binning into MAGs. Tiara is also the first tool designed to consider organellar sequences as a separate class, allowing further analyses.

324 Trained models encapsulated within Tiara scored high accuracy for validation and test dataset,

325 suggesting that models are not overfitting. Moreover, longer $k$-mers coupled with large networks 326 resulted in the best performance in both classification stages, confirming that complex neural 327 networks can better identify informative signal within DNA sequences.

328 Using a test dataset, we have shown that Tiara performs similarly to EukRep (representing the 329 current state-of-the-art) in terms of prokaryotes classification, and outperformed it in terms of 330 classification of eukaryotes with considerably lower calculation time. Tiara was trained on a much 331 larger data set than EukRep and employed neural networks, which allowed longer k-mer (6) usage 332 and performed better with more complex data. EukRep uses linear-SVMs, which are less effective 333 when dealing with multidimensional data. Crucially, Tiara correctly classifies sequences from 334 organellar genomes (up to nearly $100 \%$ of plastid sequences and $99 \%$ of mitochondrial sequences). 335 In contrast, EukRep recovered an only small portion of organellar fragments (approximately 33\%) 336 classifying them as eukaryotic ones (Table 1).

337 Analysis of the metagenomic data from the Mediterranean Sea allowed the classification and 338 reconstruction of the organellar genomes. Tiara classified most of the contigs as nuclear genomes for 339 the fraction larger than $20 \mu \mathrm{m}$, and the smallest fraction was dominated by prokaryotes, as already 340 reported in previous studies (Tully et al. 2018). Analysis of a range of $k$-mers and a sequence length 341 cut-off confirmed that the lowest false positive rate is achieved for $k$-mer 6 with a minimum 342 sequence length of $3 \mathrm{~kb}$. Even though organellar fragments constituted less than $10 \%$ of contigs, they 343 were among the longest ones. Thanks to Tiara, we reconstructed three partially complete plastid 344 genomes and twelve almost complete mitochondrial genomes from the Mediterranean Sea data set. 345 Only one plastid genome was classified to the species level ( $99 \%$ of identity); however, it most likely 346 represents a different strain than those deposited as reference data in databases. For all 347 mitochondrial genomes, the classification was restricted by the NCBI database's lack of close 348 reference. Six of the identified genomes had only a moderate similarity to crustacean genomes of 349 Undinula vulgaris ( 72\%) and Paracyclopina nana ( $\sim 6 \%)$. This result suggests that we recovered 350 mitochondrial genomes of crustacean species currently not represented in the NCBI database. 
Still, some challenges remain. The classification of shorter sequences might be wrong because those sequences are less informative. Thus, we recommend analyzing sequences that are longer than $3 \mathrm{~kb}$ to reduce the false-positive rate. The classification of eukaryotic sequences might also be disturbed

354 by the existence of NUMTs and NUPTs - fragments of mitochondrial or plastid genomes localized in 355 nuclear genomes (Kim and Lee, 2018). Those fragments might be misassigned as organellar sequences. Another problem constitutes introns and other extremely divergent non-coding regions, which might significantly disturb $k$-mer frequencies locally, resulting in the wrong prediction if a given sequence is too short to retain distinctive signal. Finally, the relatively high similarity of rDNA operons between groups can result in misclassification of those regions. Thus, we suggest using additional tools like Phyloflash to analyze those regions (Gruber-Vodicka et al., 2020).

In the current analysis, the class "unknown" from the first stage of classification has been added to the class "eukaryotic" to maximize eukaryotic sequences' recovery. This decision was based on the assumption that ambiguous predictions will less likely fall into the class prokaryote since the set of prokaryotic genomes used for training was extensive, diverse and evenly-sampled. Consequently,

365 there is a high chance that those fragments belong to eukaryotic genomes. The prokaryotic and viral

366 sequences which might end up in the class "unknown" can also be easily removed during

367 preprocessing step like binning and bin refinement. However, our assumption might slightly increase

368 the number of false positives in the first stage of classification.

369 Currently, sequences shorter than the given threshold remain unclassified and should be treated 370 separately using gene-centric approaches. Those sequences are less informative and might 371 significantly increase the number of false positives. However, it is still worth analyzing them to detect 372 mitochondrial and plastid genomes of rare protists.

373 Despite its advantages, organellar genomes so far have not been widely used, compared to 374 metabarcoding or single-cell approaches. We hope that Tiara will enable researchers to make more 375 use of metagenomic data. Organellar data can be employed for phylogenomic reconstruction and 376 uncover new eukaryotic lineages, as already have been shown for mitochondrial genomes of marine 377 heterotrophic protists (Wideman et al., 2020). Organellar sequences, similarly to barcodes, are also 378 applicable for diversity assessment and biogeographic studies. Even partial organellar genomes 379 might be successfully used to study organellar genomes' structure and content (Cuvelier et al., 2010; 380 Hovde et al., 2014). Ultimately, all these approaches enable a deeper understanding of diversity and 381 evolution of eukaryotes.

382 The reconstructed underrepresented genomes can be used to supplement existing databases. That 383 would further reduce the false positives and allow for more precise classification. Tiara could also be 
384 broadly used for metagenomic data preprocessing to remove eukaryotic contamination, including

385 more difficult to distinguish from prokaryotic data organellar sequences.

386 Our analyses have shown that publicly available metagenomic data contain a considerable number of

387 organellar DNA fragments. Among analyzed datasets, we were able to identify organellar sequences

388 of previously unreported plastid and mitochondrial genomes. Our results also suggest that even from

389 low-quality data, which are insufficient for nuclear genomes assembly, almost complete organellar

390 genomes can be reconstructed.

$391 \quad$ Acknowledgements

392 We thank Stanisław Dunin-Horkawicz and Kacper Maciszewski for critical reading of the manuscript 393 and many colleagues for carrying out beta tests of the software.

\section{Funding}

395 This work was supported by the European Molecular Biology Organization [EMBO Installation Grant 396 to AK].

\section{References}

Agarwala,R. et al. (2018) Database resources of the National Center for Biotechnology Information. Nucleic Acids Res., 46, D8-D13.

Almeida,A. et al. (2019) A new genomic blueprint of the human gut microbiota. Nature, 568, 499504.

Altschul,S.F. et al. (1990) Basic local alignment search tool. J. Mol. Biol., 215, 403-410.

Andújar,C. et al. (2015) Phylogenetic community ecology of soil biodiversity using mitochondrial metagenomics. Mol. Ecol., 24, 3603-3617.

Cock,P.J.A. et al. (2009) Biopython: freely available Python tools for computational molecular biology and bioinformatics. Bioinformatics, 25, 1422-1423.

409 Crampton-Platt,A. et al. (2016) Mitochondrial metagenomics: letting the genes out of the bottle. $410 \quad$ Gigascience, 5, 15.

411 Cuvelier,M.L. et al. (2010) Targeted metagenomics and ecology of globally important uncultured eukaryotic phytoplankton. Proc. Natl. Acad. Sci., 107, 14679-14684. 
Delmont,T.O. et al. (2020) Functional repertoire convergence of distantly related eukaryotic plankton lineages revealed by genome-resolved metagenomics.

Delmont,T.O. et al. (2015) Genome reconstructions indicate the partitioning of ecological functions inside a phytoplankton bloom in the Amundsen Sea, Antarctica. Front. Microbiol., 6.

Delmont,T.O. and Eren,A.M. (2016) Identifying contamination with advanced visualization and analysis practices: Metagenomic approaches for eukaryotic genome assemblies. PeerJ, 2016, 116.

Dröge,J. et al. (2015) Taxator-tk: precise taxonomic assignment of metagenomes by fast approximation of evolutionary neighborhoods. Bioinformatics, 31, 817-824.

Duncan,A. et al. (2020) Metagenome-assembled genomes of phytoplankton communities across the Arctic Circle. bioRxiv Microbiol., 2020.06.16.154583.

Eren,A.M. et al. (2015) Anvi'o: An advanced analysis and visualization platformfor 'omics data. PeerJ, 2015, 1-29.

Grigoriev,I. V. et al. (2012) The Genome Portal of the Department of Energy Joint Genome Institute. Assembly from Metagenomes. mSystems, $\mathbf{5}$.

Hovde,B.T. et al. (2014) The mitochondrial and chloroplast genomes of the haptophyte 604.

Keeling,P.J. and Campo,J. del (2017) Marine Protists Are Not Just Big Bacteria. Curr. Biol., 27, R541R549.

Kim,E. et al. (2011) Newly identified and diverse plastid-bearing branch on the eukaryotic tree of life. tomato. PLoS One, 13, 1-23. 
bioRxiv preprint doi: https://doi.org/10.1101/2021.02.08.430199; this version posted February $9,2021$. The copyright holder for this preprint (which was not certified by peer review) is the author/funder, who has granted bioRxiv a license to display the preprint in perpetuity. It is made available under aCC-BY-ND 4.0 International license.

Conference on Learning Representations, ICLR 2015 - Conference Track Proceedings.

442

443

444

Kopf,A. et al. (2015) The ocean sampling day consortium. Gigascience, 4, 27.

Krawczyk,P.S. et al. (2018) PlasFlow: predicting plasmid sequences in metagenomic data using genome signatures. Nucleic Acids Res., 46, e35.

Lam,S.K. et al. (2015) Numba: a LLVM-based Python JIT compiler. Proc. Second Work. LLVM Compil. Infrastruct. HPC - LLVM'15, 1-6.

Leconte,J. et al. (2020) Genome resolved biogeography of mamiellales. Genes (Basel)., 11.

Liang,Q. et al. (2020) DeepMicrobes: taxonomic classification for metagenomics with deep learning. NAR Genomics Bioinforma., 2.

Meng,G. et al. (2019) MitoZ: a toolkit for animal mitochondrial genome assembly, annotation and visualization. Nucleic Acids Res., 47, e63-e63.

Obiol,A. et al. (2020) A metagenomic assessment of microbial eukaryotic diversity in the global ocean. Mol. Ecol. Resour., 20, 718-731.

Olm,M.R. et al. (2019) Genome-resolved metagenomics of eukaryotic populations during early colonization of premature infants and in hospital rooms. Microbiome, 7, 26.

Paszke,A. et al. (2019) PyTorch: An Imperative Style, High-Performance Deep Learning Library. Adv. Neural Inf. Process. Syst., 32.

Pesant,S. et al. (2015) Open science resources for the discovery and analysis of Tara Oceans data. Sci. Data, 2, 150023.

Piganeau,G. et al. (2008) Picoeukaryotic sequences in the Sargasso Sea metagenome. Genome Biol., 9, 1-11.

Piganeau,G. and Moreau,H. (2007) Screening the Sargasso Sea metagenome for data to investigate genome evolution in Ostreococcus (Prasinophyceae, Chlorophyta). Gene, 406, 184-190.

Ren,J. et al. (2018) Alignment-Free Sequence Analysis and Applications. Annu. Rev. Biomed. Data Sci., 1, 93-114.

Richter,D. et al. (2019) Genomic evidence for global ocean plankton biogeography shaped by largescale current systems. 1-36. 
bioRxiv preprint doi: https://doi.org/10.1101/2021.02.08.430199; this version posted February $9,2021$. The copyright holder for this preprint (which was not certified by peer review) is the author/funder, who has granted bioRxiv a license to display the preprint in perpetuity. It is made available under aCC-BY-ND 4.0 International license.

Salazar,G. et al. (2019) Gene Expression Changes and Community Turnover Differentially Shape the Global Ocean Metatranscriptome. Cell, 179, 1068-1083.e21.

Schön,M.E. et al. (2020) PhyloMagnet: Fast and accurate screening of short-read meta-omics data using gene-centric phylogenetics. Bioinformatics, 36, 1718-1724.

Smith,T.F. and Waterman,M.S. (1981) Identification of common molecular subsequences. J. Mol. Biol., 147, 195-197.

Strassert,J.F.H. et al. (2018) Single cell genomics of uncultured marine alveolates shows paraphyly of basal dinoflagellates. ISME J., 12, 304-308.

Sunagawa,S. et al. (2015) Ocean plankton. Structure and function of the global ocean microbiome. Science (80-. )., 348, 1261359.

Tietz,M. et al. (2017) skorch: A scikit-learn compatible neural network library that wraps PyTorch.

de Vargas,C. et al. (2015) Ocean plankton. Eukaryotic plankton diversity in the sunlit ocean. Science (80-. )., 348, 1261605.

Varoquaux,G. and Grisel,O. (2009) Joblib: running python function as pipeline jobs. Packag. python. org/joblib.

West,P.T. et al. (2018) Genome-reconstruction for eukaryotes from complex natural microbial communities. Genome Res., 28, 569-580.

Wideman,J.G. et al. (2020) Unexpected mitochondrial genome diversity revealed by targeted singlecell genomics of heterotrophic flagellated protists. Nat. Microbiol., 5, 154-165.

Wood,D.E. et al. (2019) Improved metagenomic analysis with Kraken 2. Genome Biol., 20, 1-13. 